\title{
Relación entre la edad materna avanzada y la morbilidad neonatal en el Hospital Nacional Arzobispo Loayza. Enero -Octubre del 2012
}

Alejandro Lemor'

\section{RESUMEN}

Objetivo: Determinar si existe relación entre la edad materna avanzada y la morbilidad neonatal en el Hospital Nacional Arzobispo Loayza.

Material y Métodos: Estudio tipo cohorte, comparativo, analítico, retrospectivo. Se utilizó una muestra de 2771 recién nacidos entre Enero y Octubre del 2012, de los cuales fueron 460 de madres mayores de 35 años y 2311 de madres entre 20 y 34 años. El análisis estadístico utilizó SPSS 21.0 y se incluyó la prueba de chi cuadrado, siendo un valor de $p<0.05$ considerado para la significancia estadística.

Resultados: El 1.3\% de los recién nacidos de madres añosas presentaron trisomía 21 , en comparación con un $0.13 \%$ en las madres de 20 a 34 años ( $<$ 0.001; RR: 10.16 IC 95\%: 2.5 - 40.8). El parto por cesárea representó el $51.52 \%$ en el grupo de mayores de 35 y $37.78 \%$ en el grupo control ( $p<0.001$; RR: 1.75 , IC $95 \%: 1.43-2.14$ ). No se halló diferencia significativa al comparar el resto de morbilidades en ambos grupos, siendo estas la Enfermedad de Membrana Hialina ( $\mathrm{p}=0.824)$, Síndrome de Distrés Respiratorio $(\mathrm{p}=0.448)$, Sepsis Neonatal $(\mathrm{p}=0.309)$.

Conclusiones: La edad materna avanzada se asoció con una mayor tasa de cesáreas y más riesgo de tener un hijo con síndrome de Down. No se evidenció que la edad materna avanzada se asocie al aumento de la morbimortalidad neonatal. (Horiz Med 2014; 14(2): 22-26)

Palabras clave: ventilación mecánica, neonato, cuidado neonatal. (Fuente: DeCS BIREME).

Relationship between advanced maternal age and neonatal morbidity in the Hospital Nacional Arzobispo Loayza between January and October 2012

\begin{abstract}
Objective: Determine the relationship between advanced maternal age and neonatal morbidity in the Hospital Nacional Arzobispo Loayza.

Material and Methods: Cohort study, comparative, analytical, retrospective. A total of 2771 infants between J anuary and October 2012 were included in the study; of which 460 were born of mothers aged over 35 and 2311 of mothers between 20 and 34 years. Statistical analysis used SPSS 21.0 and included the chi-square test, considering a $p$ value of $<0.05$ for statistical significance.

Results: $1.3 \%$ of newborns of older mothers had trisomy 21 , compared with $0.13 \%$ in mothers aged 20 to 34 years $(p<0.001$, RR: $10.16,95 \% \mathrm{Cl} 2.5-40.8)$. The cesarean delivery accounted for $51.52 \%$ in the group of aged over 35 and $37.78 \%$ in the control group ( $\mathrm{p}<0.001$, RR $1.75,95 \% \mathrm{Cl} 1.43-2.14)$. There was no significant difference for other morbidities in both groups; hyaline membrane disease $(p=0.824)$, respiratory distress syndrome $(p=0.448)$ and neonatal sepsis $(p=0.309)$. Conclusions: Advanced maternal age was associated with a higher rate of $\mathrm{C}$-sections and a higher risk of having a child with Down syndrome. There was no evidence that advanced maternal age is associated with increased neonatal morbidity. (Horiz Med 2014; 14(2): 22-26)
\end{abstract}

Key words: mechanical ventilation, neonatal, neonatal care. (Source: MeSH NLM).

\footnotetext{
1 Postdoctoral Research Fellow PERFUSE y Teacher Assistant PPCR/ Harvard Medical School. USA
} 


\section{INTRODUCCIÓN}

Hoy en día, las mujeres deciden postergar la maternidad para dedicarse a una carrera profesional (1), lo que conlleva al aumento de la incidencia de gestantes de edad avanzada y a su vez, posibles complicaciones en el recién nacido.

En el Perú, todavía hay una alta incidencia de embarazos adolescentes en comparación con países desarrollados, en los cuales cada vez aumenta la edad en la que la mujer decide tener hijos.

Pero la tendencia indica que el número de gestantes con más de 35 años va ir en aumento, y es importante conocer los riesgos de postergar la maternidad hasta después de los 35 años, tanto para la madre como para el recién nacido (2)

El incremento en la incidencia de mujeres gestantes de edad avanzada, debido al deseo del mejoramiento de la mujer en edad madura, por la competitividad profesional y laboral a la cual está sometida. Se presenta una situación nueva en particular, en la última década el desarrollo de las técnicas de fertilidad asistida para las parejas que no han logrado tener hijos por diversas causas de infertilidad. Esto hace que la búsqueda de un hijo, en muchos casos el primero, esté diferida hasta edades mayores de los 35 años.

Varios estudios demuestran la relación entre la edad materna y mayores riesgos de enfermedad en el embarazo y en el neonato (3-8). Entre las principales morbilidadesasociadas, se incluye: prematuridad, baj 0 peso al nacer, parto por cesárea, depresión neonatal a los 5 minutos(Apgar a los 5 minutos) y en menor proporción a sepsis neonatal, distres respiratorio y enfermedad de membrana hialina.

En esta investigación, se tuvo como objetivo analizar los datos de recién nacidos de madres mayores de 35 años.

\section{MATERIAL Y MÉTODOS}

Diseño de tipo observacional, cualitativo, analítico, cohorte retrospectivo, tomando como población a todos los neonatos nacidos de madre mayor $\mathrm{y} / \mathrm{o}$ igual a 35 años y madres entre 20 a 34 años, en el Servicio de Neonatología del Hospital Nacional Arzobispo Loayza, desde Enero a Octubre de 2012. Se revisaron las Historias clínicas y se registraron los datos.

La población de recién nacidos entre Enero y Octubre del 2012 fue de 3450 pacientes. La muestra estuvo conformada por 2771, de los cuales: 2311fueron recién nacidos de madres entre 20 y 34 años y los otros 460 restantes, fueron de madres mayores de 35 años.

Las variables que se utilizaron fueron cualitativas, se incluyó: edad materna, control prenatal (CPN), tipo de parto, Apgar a los 5 minutos, edad gestacional, adecuación, síndrome de distres respiratorio, enfermedad de membrana hialina, sepsis neonatal y trisomia 21. Se utilizó el programa estadístico SPSS 21.0 para analizar los datos y la prueba de Chi cuadrado para determinar la relación entre variables y determinar su significancia estadística.

\section{RESULTADOS}

Entre Enero y Octubre del 2012, hubo 460 recién nacidos de madres mayores de 35 años, lo que correspondió al $13.33 \%$ del total de recién nacidos en el HNAL en ese periodo de tiempo. Siendo el $70 \%$ recién nacidos de madres entre 20 y 34 años y un $16.67 \%$ los recién nacidos de madres menores de 20 años.

En las madres mayores de 35 años, la media de edad fue de 37.8 años, el $72.17 \%$ (332) tuvieron un adecuado CPN y en el $51.52 \%$ (237) el tipo de parto fue por cesárea. En comparación con un total de 2311 recién nacidos de madres entre 20 y 34 años, con una media de edad de 26 años, el 73.73 \% (1704) tuvieron un CPN adecuado y el $37.78 \%$ (873) la vía de termino del embarazo fue por cesárea.

36 de los recién nacidos de madres mayores de 35 años fueron pretérmino (7.83\%), mientras que de los recién nacidos de madres entre 20 y 34 años, fue de $11.03 \%(255 \mathrm{RN})$. Los recién nacidos pequeños para 
edad gestacional fueron 41 , conformando un $8.91 \%$ en las madres añosas y un $9.17 \%$ (212 RN) en las madres entre 20 y 34 años. El 1.96\% (9 RN) de los hijos de madres añosas tuvieron un Apgar menor de 7 a los 5 minutos, en comparación con 1.08\% ( $25 \mathrm{RN}$ ) de los hij os de madres entre 20 y 34 años.
Los hij os de madres añosas que nacieron con síndrome de distresrespiratorio fueron6, el $1.3 \%$ y de madres entre 20 y 34 fueron 22 , el $0.95 \%$. Cinco, de los RN de madres añosas (1.09\%), presentaron enfermedad de membrana hialina, en comparación con el 1.38\% (32) de RN de madres entre 20 y 34 años. Un 3.04\%, 14 de losRN de madres añosas, tuvieron sepsis neonatal y seis RN, el 1.30\% nacieron con trisomia 21. De los hijos de madres entre 20 y 34 años, 51 (2.21\%) presentaron sepsis al nacer y 3, nacieron con trisomia 21 .

Tabla 1. Características demográficas de la población en estudio.

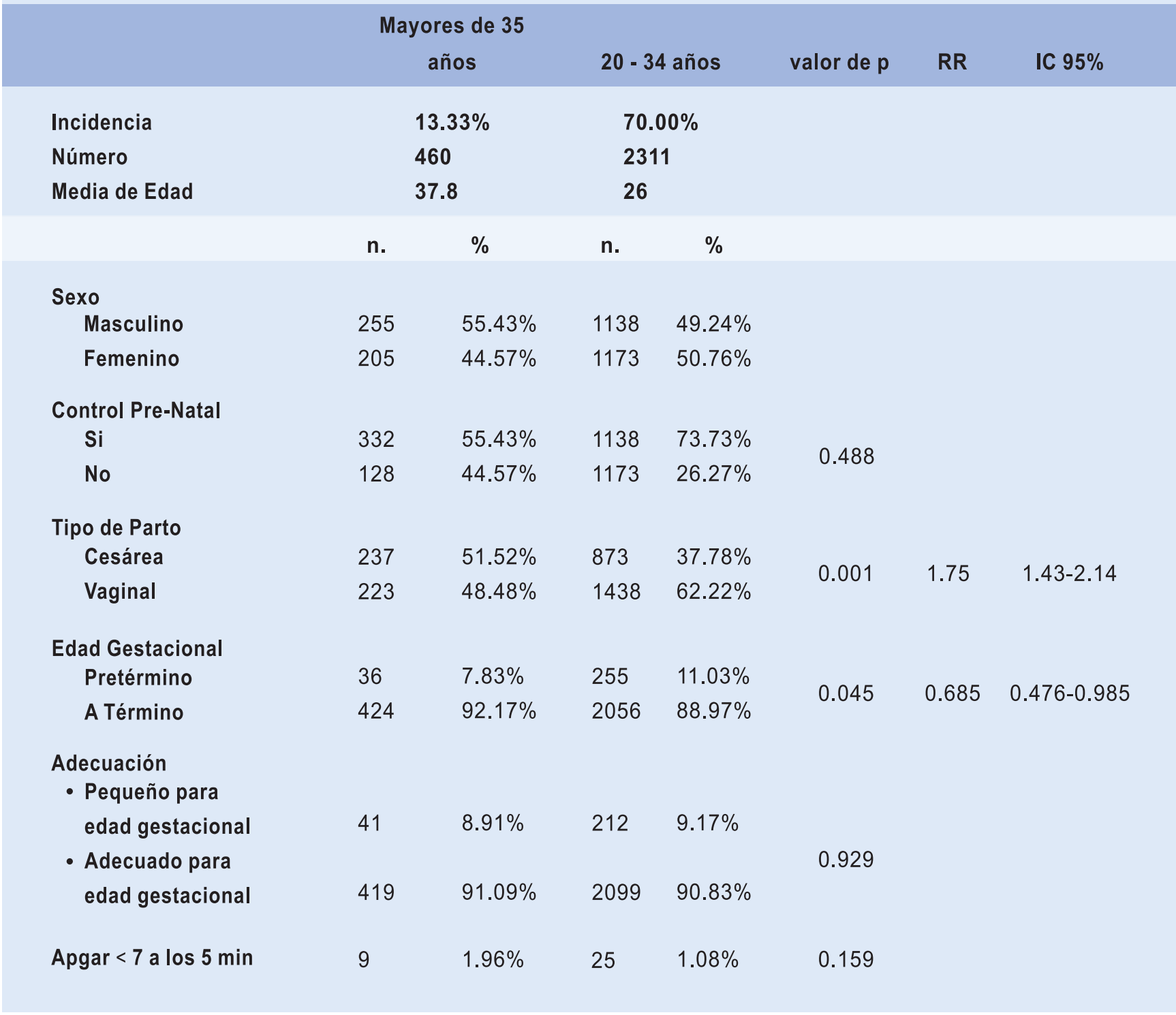




\section{DISCUSIÓN}

La incidencia de nacimientos en gestantes añosas del HNAL fue de $13.3 \%$, siendo ésta menor a $16 \%$, cifra estimada por CELADE/CEPAL (9) que correspondió a nacimientos en el Perú de madres mayores de 35 años entre el 2005 y 2010.Según la ONU, la edad media de fecundidad en el Perú ha ido en aumento, en 1995 fue de 28.56 años, en el 2010 de 28.85 años, y se estima que para el 2015 será de 29 años, lo que significará un aumento en la incidencia de nacimientos de madres añosas en un futuro muy cercano (2).

Coincidimos con varios estudios que demuestran que un recién nacido de madre mayor de 35 años tiene mayor riesgo de presentar anomalías cromosómicas, entre ellas, la más común el síndrome de Down (1017).

Nosotros, evidenciamos que el $1.3 \%$ de los RN de madres añosas presentaron trisomía 21 , en comparación con un $0.13 \%$ en las madres de 20 a 34 años, siendo significativamente estadístico, con un valor $p$ de 0.001 .

Siendo las madres mayores de 35 años las que presentaron mayor riesgo de tener un hijo con síndrome de Down (RR: 10.16 IC 95\%: 2.5 - 40.8). Sin embargo; se necesita una muestra más amplia para poder determinar el riesgo, ya que al tener un intervalo de confianza muy amplio, este pierde significancia. No se encontró otro tipo de cromosopatias en la población estudiada, quizá por el tamaño de muestra.

El parto por cesárea, predominó en las madres mayores de 35 años, siendo $51.52 \%$ del total, en comparación con madres de 20 a 34 años con $37.78 \%$ lo que fue estadísticamente significativo $(\mathrm{p}<0.001)$ con un RR: 1.75 (IC 95\%: 1.43 - 2.14).

Con respecto al CPN, ambos grupos se encuentran por encima del porcentaje de cobertura de gestantes controladas según el MINSA para Enero a Setiembre de 2012 , siendo dicho valor de $56.4 \%$ para Lima (10), y en el HNAL se encontró que ambos grupos tuvieron CPN en más del 70\%. Debiendo considerar que dicho hospital es de nivel III-1 y presenta un nivel de atención superior a otros centros de salud.
Contrariamente a lo descrito por distintos autores $(8,10)$, en este estudio no se encontró asociación entre edad materna avanzada (EMA) y parto pretérmino, siendo $7.83 \%$ en las madres mayores de 35 años y de $11.03 \%$ en las madres de 20 a 34 años, con un valor $\mathrm{p}$ de 0.045 y un RR de 0.685 (IC 95\%: $0.476-0.985$ ). Al analizar los CPN, en ambos grupos no se halló diferencias, lo que podría, en parte, explicar que la variable de EMA no influyó sobre la posibilidad de tener un RN prematuro.

Torres en el 2009, en un estudio descriptivo retrospectivo en el hospital de Maternidad de El Salvador (18) y Schuller et al en el 2007, estudiando a 436 pacientes embarazadas atendidas en el Servicio de Obstetricia del Hospital Universitario de Caracas (7), concluyeron que la mujer embarazada mayor de 35 años no presenta mayor riesgo de complicaciones obstétricas y perinatales en dichos nosocomios. También en este estudio, no se demostró significancia estadística para concluir que las madres mayores de 35 años presenten un mayor riesgo para enfermedades neonatales como Enfermedad de Membrana Hialina $(p=0.824)$, Síndrome de Distres Respiratorio $(p=0.448)$, Sepsis Neonatal $(p=0.309)$, PEG o parto pretermino. A pesar de ser las gestantes de edad avanzada un grupo de alto riesgo, el control adecuado y frecuente para la detección oportuna de las complicaciones, y al ser atendidas en un hospital III-1 como el HNAL, podrían reducir el riesgo de este grupo etario para tener complicaciones neonatales (19-23).

La media de edad para las madres mayores de 35 años, fue de 37.8 , lo que nos podría explicar porque no hay gran diferencia entre ambos grupos ya que la mayoría de este grupo se acerca al corte de 35 años.

En conclusión, en el presente estudio, se encontró asociación entre el parto por cesárea y la EMA (mayor de 35 años).Con una incidencia de trisomía 21 mayor.

No se evidenció que la edad materna avanzada se asocie con aumento de la morbimortalidad neonatal en la población estudiada.

Se recomienda estudiar a una mayor población para poder establecer mayor asociación entre la edad materna avanzada y la morbilidad neonatal. 


\section{Fuentes de financiamiento}

El estudio ha sido autofinanciado por el autor.

\section{Conflicto de interés}

El autor declara no tener ningún conflicto de interés.

\section{Correspondencia:}

Alejandro Lemor

Dirección: 75 Saint Alphonsus Apt 1107 Boston MA

Teléfono: +1 6176327760

Correo electrónico: alemor@bidmc.harvard.edu

\section{REFERENCIAS BIBLIOGRÁFICAS}

1. Paredes N. Maternidad postergada. HorizMed 2013; 13(1):45-50

2. UN. Department of Economic and Social Affairs. Population Division, Population Estimates and Projections Section. World Population Prospects, the 2010 Revision. Fertility and Mean Age of Childbearing. http:/ / esa.un.org/ wpp/ Excel-Data/ fertility.htm

3. Ramírez C, NazerJ, Cifuentes L, Águila A. Cambios en la distribución etaria de las madres en Chile y en el Hospital Clínico de la Universidad de Chile y su influencia en la morbimortalidad neonatal. Rev Chil Obstet Ginecol 2012; 77(3): 183 - 189.

4. Tipiani-Rodríguez O. ¿Es la edad materna avanzada un factor de riesgo independiente para complicaciones materno-perinatales?. Rev Per Ginecol Obstet 2006;52(3):89-99.

5. Brian T, Bateman Lynn L. Simpson. Higher rate of stillbirth at the extremes of reproductive age: A large nation wide sample of deliveries in the United States. Am J of Obstet and Gynecol 2006.

6. Froen J, Arnestad M, Frey K, et al. Risk factors for sudden intrauterine unexplained death: epidemiologic characteristic of singleton cases in Oslo, Norway 1986-1995. Am J of Obstet and Gynecol 2001; 184:694-702.

7. SchullerA, Benítez-Guerra G, Estudio de las gestantes de edad avanzada en el Hospital Universitario de Caracas. Rev de la Facultad de Medicina 2007; 30(1).

8. Nolasco-Blé A, Hernández-Herrera R, Ramos-González R. Hallazgos perinatales de embarazos en edad materna avanzada. Ginecol Obstet Mex 2012; 80(4):270-275.

9. Naciones Unidas. Centro Latinoamericano y Caribeño de Demografía (CELADE), División de Población de la Comisión Económica para América Latina y El Caribe (CEPAL). http://celade.cepal.org/ redatam/index. es. html

10. MINSA, Oficina de Estadística. Reporte Mensual de la Producción de Servicios de Salud Materno Perinatal. Cobertura de Gestantes Controladas Enero-Setiembre; 2012.
11. Villa R, Candenas M, Alvarez FV. Combinación de la alfafetoproteína sérica, gonadotrofina coriónica y edad materna para el cálculo de riesgo de Síndrome de Down en el embarazo. Prog Diagn Pren 1992; $4(3)$.

12. Chamy V, Cardemil F, Betancour P, Ríos M, Leighton L. Riesgo obstétrico y perinatal en embarazadas mayores de 35 años. Rev Chil Obstet Ginecol 2009;74:331-8.

13. Reefhuis J, Honein M. Maternal age and chromosomal birth defects. Atlanta 1998-2000: teenager or thirty-something, who is the risk?. Birth Defects Res A Clin Mol Teratol 2004; 70:572-9.

14. Sherman SL, Freeman SB, Allen EG, Lamb NE. Risk factors for non disjunction of trisomy 21. Citogenetic Genome Res 2005;111:27380 .

15. Nazer J, Cifuentes L, Águila A, Ureta P, Bello M, Correa F, et al. Edad materna y malformaciones congénitas: Un registro de 35 años. 1970-2005. Rev Méd Chile 2007 Nov; 135(11):1463-1469.

16. Bianco A, Stone J, Lynch L, Lapinsky K, Berkowitz G. Pregnancy outcome at age 40 and older.ObstetGinecol 2003; 87: 917.

17. Chamy V, Cardemil F, BetancourP, Ríos M, LeightonL. Riesgo obstétrico y perinatal en embarazadas mayores de 35 años. Rev Chil Obstet Ginecol 2009; 74(6): 331 - 338.

18. Torres $C$. Resultado perinatal y obstétrico en embarazadas mayores de 35 años en el hospital nacional de maternidad en el periodo de enero a diciembre de 2009. Tesis para grado de Ginecólogo Obstetra, Universidad de El Salvador 2009.

19. González-Garay A. Actualidades sobre la taquipnea transitoria del recién nacido. Acta Pediatr Mex 2011; 32(2):128-129.

20. Colomé C, Carrasco M, Agramunt S, Checa M, Carreras R. Fertilidad en mujeres mayores de 40 años. Barcelona Ginecología y Obstetricia Clínica 2008;9(4):216-227.

21. González C, Omaña Alonso. Protocolos de Neonatología. Síndrome de distrés respiratorio neonatal o enfermedad de membrana hialina. Bol Pediatr 2006; 46(1): 160-165.

22. Ceriani JM. Programa de Actualización en Neonatología (PRONEO). Buenos Aires. Editorial Panamericana; 2000: Módulo 1: 113-162.

23. Cabrera JH. Resultados perinatales y maternos de los embarazos en edad madura. Rev Cub Obstet y Ginecol 2003; (29): 28-9. 